eISSN 2444-7986

DOI: http://dx.doi.org/10.14201/orl201673.14446

Artículo de revisión

\title{
UNA SOLA MÉTRICA NO CUENTA TODA LA HISTORIA DE LA PRODUCCIÓN CIENTÍFICA. III. GESTIÓN Y CONCLUSIONES
}

\section{One metric does not tell the whole story of scientific production. III. Management and conclusions} \author{
España. \\ Correspondencia: nievesglez@gmail.com
}

Nieves GONZÁLEZ-FERNÁNDEZ-VILLAVICENCIO

Universidad de Sevilla. Directora de la Biblioteca de la Facultad de Ciencias Económicas y Empresariales. Sevilla.

Fecha de recepción: 30 de abril de 2016

Fecha de aceptación: 22 de mayo de 2016

Fecha de Publicación: 10 de junio de 2016

Conflicto de intereses: Los autores declaran no tener conflictos de intereses

Imágenes: Los autores declaran haber obtenido las imágenes con el permiso de los pacientes

Política de derechos y autoarchivo: se permite el autoarchivo de la versión post-print (SHERPA/RoMEO)

Licencia CC BY-NC-ND. Licencia Creative Commons Atribución-NoComercial-SinDerivar 4.0 Internacional

(C) Universidad de Salamanca. Su comercialización está sujeta al permiso del editor

RESUMEN

\begin{abstract}
Introducción: Existen diferentes estrategias de publicación científica que pueden ser abordadas desde la perspectiva de la gestión, de cara a la evaluación por las agencias nacionales. Aparición de las métricas alternativas, servicios y proveedores. Método: Revisión narrativa. Resultados: Se presentan los rasgos característicos de los criterios de evaluación de las publicaciones científicas por las agencias de evaluación españolas y se describen los servicios altmétricos. Conclusiones: Se plantea la necesidad de contar con sistemas nacionales de evaluación de la producción científica para evitar el sesgo actual y el desconocimiento del sector editorial del que adolecen WoS y Scopus. Y también la necesidad de contar con otros sistemas altmétricos, complementarios a los tradicionales, que amplíen el tipo de productos científicos y evalúen el impacto científico social.
\end{abstract}

PALABRAS CLAVE gestión de la información; publicación científica; visibilidad web; impacto científico; métrica; calidad

SUMMARY

\begin{abstract}
Introduction: There are different strategies for scientific publication that can be addressed from the perspective of management, ahead of the assessment by national agencies. Rising of alternative metrics, services and suppliers. Method: Narrative review. Results: the characteristic features of the evaluation criteria of scientific publications by Spanish agencies evaluation are presented and described altmetrics services. Conclusions: The need for national evaluation systems of scientific production to avoid the current bias and ignorance of the publishing sector which suffer WoS and Scopus arises. And also the need for other altmetric systems, complementary to traditional, to expand the type of scientific products and evaluate the scientific social impact.
\end{abstract}




\section{INTRODUCCIÓN}

ESTRATEGIAS DE GESTIÓN. En los dos artículos anteriores de esta serie hemos realizado un recorrido por las estrategias de publicación científica que deben tener en cuenta los investigadores españoles de cara a su visibilidad y las relacionadas con la evidencia de calidad de sus publicaciones. En este artículo abordaremos estas estrategias desde una perspectiva de gestión y veremos qué aspectos de los contemplados valoran hasta hoy las agencias de evaluación para las acreditaciones y los sexenios.

Ya sabemos que la base de la evaluación hoy en día es el artículo científico y, por lo tanto, sólo aquellas revistas que cumplan con los criterios de las agencias evaluadoras (ANECA y su CNEAl) serán más solicitadas por los autores españoles, así como las editoriales de libros en segundo lugar.

Para Giménez-Toledo [1], los criterios que definen estos organismos de evaluación se basan por un lado en cuestiones relacionadas con la política científica del país y las prioridades que establecen, las prácticas internacionales de evaluación $y$, por último, en los trabajos desarrollados por distintos grupos de investigación españoles, que crearon los indicadores de revistas científicas de la ANECA, los de revistas evaluables de CNEAI y los criterios del sello de calidad FECYT (Grupo EC3 de la Universidad de Granada y Grupo EPUC del CSIC).

Los criterios de las agencias de evaluación han ido evolucionando a lo largo del tiempo desde aquellos más restrictivos de las revistas de la WoS hasta otros criterios incluso diferenciados por áreas.

Siguiendo con Giménez-Toledo [1], los rasgos característicos de los criterios de evaluación de las publicaciones científicas en los que coinciden las agencias de evaluación españolas son los siguientes:

1. Proceso de revisión por pares, basándose en el juicio de expertos de cada panel temático e indicadores bibliométricos.

2. Criterios de evaluación diferenciados por disciplinas - no es lo mismo Biomedicina que Historia-.

3. La evaluación es sobre el canal, no el contenido. El autor puede hacer una defensa del mismo en el proceso de evaluación.
4. Los artículos de revista tienen más peso que los libros o capítulos de libros que se publican en Humanidades y Sociales. La equivalencia sería un libro igual a dos artículos o un artículo y un capítulo.

5. Apenas hay fuentes que aporten valores para los indicadores de calidad de los libros.

6. En la evaluación de revistas, el estar en la WoS y tener factor de impacto JCR son clave en el proceso de evaluación en España.

7. En el caso de no tener estos indicadores pueden considerarse otras fuentes nacionales e internacionales. Para Humanidades y Sociales: ERIH PLUS y Scopus, aunque los indicadores de Scopus (SJR) no son considerados de forma explícita por las agencias de evaluación. Otras fuentes que aportaban indicadores para las revistas españolas y eran citadas por las agencias, han dejado de estar operativas por falta de financiación (DICE o In-RECS).

8. A pesar de que la Ley de la Ciencia apuesta por el acceso abierto, la evaluación científica se basa en fuentes cerradas. Tan sólo un $10,6 \%$ de las revistas en JCR son de acceso abierto. Las mejor valoradas no son de acceso abierto o son híbridas y el investigador por lo tanto debe optar por publicar en una revista en acceso abierto que no cobre por publicar, garantizando la disponibilidad de sus publicaciones pero perjudicándose en la evaluación; publicar en una revista en la que pague por publicar en abierto, por lo que parte de los recursos se irán a grandes grupos editoriales; o en una revista cerrada, recogida en JCR y que facilita su evaluación.

Aunque las fuentes internacionales que se imponen son la WoS con sus limitaciones y Scopus que no deja de considerarse fuente secundaria, no representan la totalidad de la producción científica que se genera en todas las partes del mundo y en distintas disciplinas. No hay que olvidar que en base a estas fuentes se organizan los rankings de universidades y los gobiernos extraen datos para comparar entre sí sus indicadores. 
Esta situación ha llevado a que determinadas regiones o países se hayan planteado crear sistemas alternativos con indicadores propios de la producción científica, al margen de la WoS o Scopus. Este es el caso de Europa con ERIH Plus (European Reference Index for the Humanities and Social Sciences), Latindex para América Latina, Europa y Portugal, RESH o DICE, SciELO así como índices de impacto nacionales como In-RECS, In-RECJ e In-RECH. En este grupo de los otros sistemas, encontramos MIAR o CIRC en España y Google Académico, que no tiene carácter de cerrado y del que se pueden obtener indicadores de revistas como hace EC3Metrics [1]. Estos casos muestran la necesidad de contar con sistemas nacionales de evaluación de la producción científica para evitar el sesgo actual y el desconocimiento del sector editorial del que adolecen WoS y Scopus. Y también la necesidad de contar con otros sistemas alternativos que amplíen el tipo de productos científicos y evalúen el impacto científico social.

Por su parte el uso de las métricas alternativas se va extendiendo en las evaluaciones del profesorado universitario. Torres Salinas decía en 2013 que ni la ANECA ni la CNEAl iban a hacer caso de estas métricas ${ }^{1}$, sin embargo hoy día vemos cómo se va extendiendo la valoración de estas métricas y su consideración en los procesos de evaluación de la producción científica para la financiación, las acreditaciones y sexenios.

\section{LAS MÉTRICAS ALTERNATIVAS}

A lo largo de este artículo hemos ido viendo distintos aspectos de las métricas alternativas o altmetrics. "Las métricas alternativas surgen en un momento en que tanto autores como editores empiezan a comprender que las métricas tradicionales no son suficientes para conocer el interés que despiertan sus publicaciones» [2]. Estas herramientas están captando diferentes tipos de impacto, pudiendo dar idea no sólo del interés que despierta en el mundo académico sino del impacto social que está teniendo el artículo. Se habla de impacto social, impacto educativo y engagement público y resultados [3].

$1 \mathrm{http}: / /$ es.slideshare.net/torressalinas/altmetrics-
indicadores-utilidades-y-limitaciones
Existen tres principales herramientas que recopilan datos altmétricos de muy diferentes fuentes, los cuales pueden ser difundidos a través de APls públicas que ponen a disposición de los demás agentes implicados: Altmetric.com, ImpactStory y PLUM Analytics (PlumX), este último servicio de pago propiedad de EBSCO que proporciona datos altmétricos de una gran cantidad de fuentes y se comercializa para universidades e instituciones de investigación. Existen muchos ejemplos del uso de altmetrics en el área de la Medicina ${ }^{2}$.

Public Library of Science (PLOS) fue el primer editor en proporcionar datos altmétricos de sus publicaciones a través de sus «articlelevel-metrics» (ALMs). Le han seguido otros grandes editores como el grupo editorial $\mathrm{Na}$ ture, Springer, Elsevier (incorpora en su base de datos Scopus el API de Altmetrics.com y ofrece datos relativos a Mendeley, ahora de su propiedad) y más recientemente Wiley. Faculty of 1000 (F1000) ${ }^{3}$ es un portal de expertos que seleccionan artículos de calidad de Medicina y Biología e incorpora datos altmétricos.

Servicios gratuitos como KUDOS ${ }^{4}$, que se puso en marcha a final de abril de 2014, pretenden ayudar a los investigadores y sus instituciones a maximizar la visibilidad e impacto de sus artículos científicos publicados, incluyendo indicadores de descargas, citaciones y altmétricos. En este producto participan más de 15 editores de suscripción, Elsevier, OECD, Bioscientifica, Bone\&Joint, Cambridge University Press, Emerald y otros.

Independientemente del proveedor de los datos altmétricos, las métricas que se utilizan se pueden clasificar en métricas a nivel de contribución individual o artículo, publicación o revista, autor e institución o grupo [4]. Las métricas a nivel de contribución individual se clasifican en métricas de uso (clics y visitas, descargas) métricas de captura (marcadores, favoritos, lectores), menciones (en blogs, comentarios, revisiones) y métricas de medios sociales («me gusta», «compartidos», «RT»). También hay que contabilizar el donuts de Altmetric y los porcentajes de ImpactStory.

- A nivel de publicación podemos considerar las métricas de PLOS.

2 https://elsevier.altmetric.com/details/2845076

$3 \mathrm{http}: / / \mathrm{f} 1000 . \mathrm{com} /$

4 https://www.growkudos.com/ 
- A nivel de autor, los perfiles de ImpactStory la rueda de PlumX, el score de ResearchGate.

- A nivel de institución, las métricas de grupo de PlumX, Altmetric para institución y Snowball Metrics.

Podemos ver un ejemplo del uso del donuts de Altmetric.com en la figura 1 , de un artículo de Nature ${ }^{5}$. El artículo "Public health: The toxic truth about sugar», de 2012, ha sido tuiteado 1749 veces y aparece en 18 noticias, 51 post de Facebook, 19 de Google+ y en 25 blogs. Aparece también en un vídeo y una entrada en Wikipedia. En Mendeley lo han incluido 454 lectores y 6 en CiteUlike. Sin duda este artículo tiene un impacto social que debe ser contado.

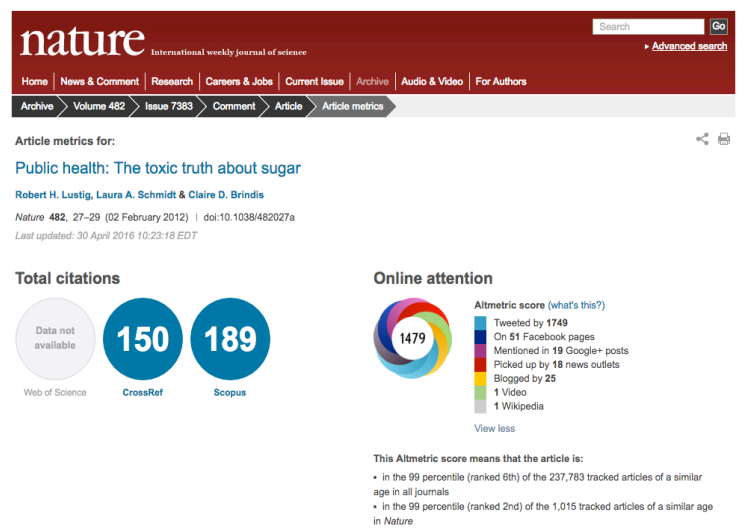

Figura 1. Ejemplo de uso de Altmetric.com en un artículo de la revista Nature.

\section{CONCLUSIONES}

La evaluación oficial de la producción científica de los investigadores, según agencias evaluadoras y diversas administraciones, se rige por una serie de métricas tradicionales basadas principalmente en los indicadores del Journal Citation Reports (JCR) de Thomson Reuters, como el factor de impacto de las publicaciones -fundamentalmente revistasy el índice de citas de las contribuciones individuales de los autores, es decir, los artículos.

\footnotetext{
${ }^{5}$ http://www.nature.com/nature/journal/v482/n7383/482027 a/metrics

6 Véanse las recomendaciones de la NISO sobre las métricas alternativas
}

En posición secundaria aparecen los indicadores de Scopus, y en los siguientes escalones productos más locales que intentan reducir el sesgo de los grandes proveedores de indicadores científicos.

A lo largo de este artículo se han visto los pasos que un investigador tipo tendría que recorrer hasta publicar en revistas de impacto de su área temática y obtener de esta forma indicadores de calidad de su producción científica que recibieran el visto bueno de las agencias de evaluación españolas, para seguir avanzando en su carrera investigadora.

Además de la necesidad de potenciar la existencia de agencias y productos de evaluación que contemplen las características nacionales o regionales de la investigación y sobre todo de áreas como las Humanidades y Ciencias Sociales, la aparición de métricas complementarias a las tradicionales es ya una realidad. Los datos están ahí. Sólo falta una mayor transparencia de los mismos como indica el informe de la $\mathrm{NISO}^{6}$, aunque desde algunos de los proveedores de datos altmétricos ya se está respondiendo a esta cuestión ${ }^{7}$. Y falta también una mayor difusión de las ventajas e inconvenientes que plantean. Pero ya están aquí.

\section{BIBLIOGRAFÍA}

1. Giménez-Toledo E. La evaluación de la producción científica: breve análisis crítico. Reli - Rev Electrónica Investig y Evaluación Educ [Internet]. 2015;21(1):26-8. Disponible en: https://ojs.uv.es/index.php/RELIEVE/arti cle/view/5160.

2. González-Fernández-Villavicencio N, Calderón-Rehecho A, DomínguezAroca MI, García-Hernández P. ¿Qué papel juegan los bibliotecarios en las altmetrics? An Doc Rev Bibl y Doc. 2015;18:1-19.

3. Konkiel $\mathrm{S}$, Sugimoto $\mathrm{CR}$, Williams $\mathrm{S}$. The use of Altmetrics in promotion and tenure. Educ Rev. 2016;2:54-5.

\footnotetext{
http://www.niso.org/topics/tt/altmetrics_initiative/

${ }^{7}$ http://plumanalytics.com/wp-

content/uploads/2015/06/NISO-Self-Reporting-Table-

Plum-Analytics-March-31-2016.pdf
} 
UNA SOLA MÉTRICA NO CUENTA TODA LA HISTORIA DE LA PRODUCCIÓN CIENTÍFICA. III. GESTIÓN Y CONCLUSIONES GONZÁLEZ-FERNÁNDEZ-VILLAVICENCIO N

4. Roemer RC, Borchardt R. Meaningful metrics: A 21st century librarian's guide to bibliometrics, altmetrics, and research impact [Internet]. Chicago, Illinois: Association of College and Research Libraries; 2015.

Disponible

en: http://www.ala.org/acrl/sites/ala.org.acrl/file s/content/publications/booksanddigitalreso urces/digital/9780838987568_metrics_OA.p df. 